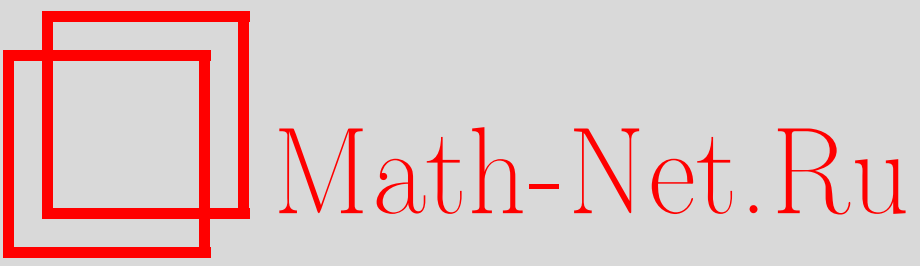

М. Н. Саушкин, О. С. Афанасьева, Е. А. Просвиркина, Оценка релаксации остаточных напряжений в упрочнённой вращающейся лопатке при ползучести, Вестн. Сам. гос. техн. ун-та. Сер. Физ.-мат. науки, 2007, выпуск 1(), 62-70

DOI: https://doi.org/10.14498/vsgtu489

Использование Общероссийского математического портала Math-Net.Ru подразумевает, что вы прочитали и согласны с пользовательским соглашением

http: //www. mathnet.ru/rus/agreement

Параметры загрузки:

IP : 35.174 .16 .151

26 апреля 2023 г., 13:01:00 


\title{
ОЦЕНКА РЕЛАКСАЦИИ ОСТАТОЧНЫХ НАПРЯЖЕНИЙ В УПРОЧНЁННОЙ ВРАЩАЮЩЕЙСЯ ЛОПАТКЕ ПРИ ПОЛЗУЧЕСТИ
}

\begin{abstract}
Разработан метод расчёта и оченки остаточных напряжений в поверхностно упрочнённом слое вращающейся лопатки при ползучести, в основе которого лежит гипотеза, согласно которой упрочнённый слой не влияет на жёсткость конструкции (играет роль тонкой «плёнки», наклеенной на его поверхность) и деформируется вместе с ним под действием внешних нагрузок в режиме «жёсткого» нагружения. В силу этого предположения расчёт кинетики релаксации остаточных напряжений в поверхностном слое сводится к склеиванию решений двух краевых задач.

В прочессе решения первой краевой задачи определяется напряжённо-деформированное состояние вращающейся лопатки при ползучести без учёта поверхностного упрочнённого слоя. Во второй краевой задаче исследуется релаксация остаточных напряжений в поверхностно упрочнённом слое, при этом слой считается единым иельм, деформирующимся в режиме «жёсткого» нагружения при заданных значениях компонент тензоров деформаций, которые определяются из решения первой краевой задачи.
\end{abstract}

Введение. Проблема увеличения времени эксплуатации конструктивных элементов приводит к развитию методов повышения долговечности деталей машин. Одним из таких методов является метод наведения сжимающих остаточных напряжений в поверхностном слое. В процессе эксплуатации происходит уменьшение по модулю остаточных напряжений. В связи с этим, весьма актуальной становится оценка времени эффективности наведённых остаточных напряжений, что особенно важно при переходе на эксплуатацию и ремонт «по техническому состоянию».

В работе предлагается обобщение метода, разработанного в работах [1-4], на случай оценки релаксации остаточных напряжений в упрочнённом слое лопатки газотурбинного двигателя (ГТД) под действием центробежных массовых сил в условиях ползучести. В основе метода лежит гипотеза, согласно которой упрочнённый слой не влияет на жёсткость конструкции (играет роль тонкой «плёнки», наклеенной на его поверхность) и деформируется вместе с ней под действием внешних нагрузок в режиме «жёсткого» нагружения. В силу этой гипотезы основная задача разбивается на три самостоятельные: 1) восстановление начального напряжённо-деформированного состояния в поверхностно упрочнённом слое по одной экспериментально замеренной компоненте тензора остаточных напряжений по толщине слоя; 2) расчёт напряжённодеформированного состояния всей конструкции при ползучести без учёта упрочнённого слоя; 3) расчёт кинетики остаточных напряжений в поверхностном слое в режиме «жёсткого» нагружения (при заданных значениях компонент тензора деформаций на поверхности элемента конструкции, которые определяются из второй краевой задачи).

Первая задача приводится к системе дифференциальных уравнений, которую можно рассматривать как относительно остаточных напряжений, так и как относительно остаточных пластических деформаций. В случае, если систему уравнений рассматривать относительно остаточных напряжений, то она при заданных пластических деформациях имеет единственное решение $[5,6]$. Полученную систему дифференциальных уравнений можно рассматривать и как обратную краевую задачу определения пластических деформаций по известным остаточным напряжениям. Данная задача при заданных остаточных напряжениях может иметь бесконечное число решений (кинематически допустимых деформаций). Для получения единственного решения необходимо вводить дополнительные гипотезы на вид тензора пластических деформаций, либо использовать экстремальные вариационные принципы для остаточных напряжений и деформаций [5-7]. В настоящей работе для решения первой задачи вводится гипотеза, согласно которой считается, что на бесконечно малом элементе криволинейной поверхности пластические деформации наводятся как на полуплоскости.

Вторая задача решается классическими методами, при этом поверхностный слой считается отсутствующим. Для решения третьей задачи поверхностный слой считается единым целым с конструкцией, деформирующимся с ней в режиме «жёсткого» нагружения.

1. Общий метод расчёта релаксации остаточных напряжений в поверхностно упрочнённом слое вращающихся стержней переменного сечения при ползучести. Одним из важных конструктивных элементов во многих технических установках является вращающийся стержень переменного сечения (вообще говоря, произвольной формы) с малой пространствен- 
ной кривизной оси. Типичными представителями такого рода конструктивных элементов являются закрученная или незакрученная лопатки газотурбинного двигателя (ГТД), посаженные на диск ГТД, площадь поперечного сечения которых (в общем случае) существенно изменяется от корневого сечения (места крепления лопатки к вращающемуся диску) до хвостового сечения (сечение, дальнее от корневого). На рис. 1 схематически представлены прямолинейная (незакрученная) лопатка с постоянной по длине площадью поперечного сечения (рис. 1, $a$ ), незакрученная (рис. 1, б) и закрученная (рис. 1,8$)$ лопатки с переменной площадью поперечного сечения от корневого до хвостового сечения, при этом для закрученной лопатки хвостовое сечений $B$ повернуто относительно корневого сечения $A$ в плоскости, перпендикулярной оси лопатки. Величина поворота зависит от конструктивных особенностей ГТД и может составлять $5^{\circ}-45^{\circ}$. Поэтому величина пространственной кривизны вдоль оси

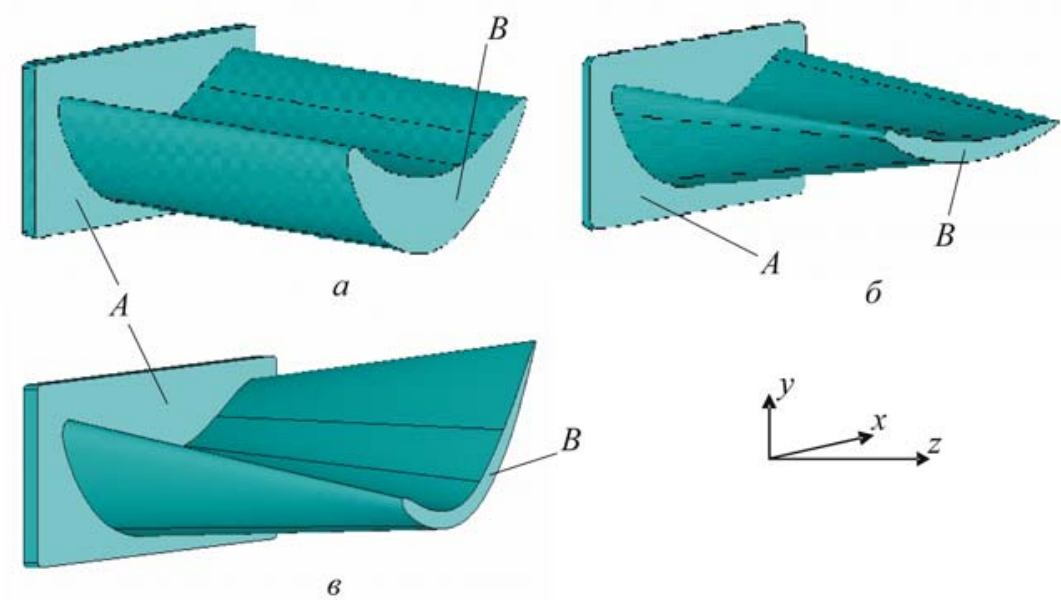

Р и с. 1. Схематический вид вращающихся лопаток лопатки незначительна.

Хорошо известно, что расчёт напряжённо-деформированного состояния и разрушения элементов конструкций с учётом нелинейного реологического деформирования прямыми численными методами оказывается очень сложным, даже при использовании современных ЭВМ. Поэтому постоянно делаются попытки найти новые подходы к решению краевых задач, с помощью которых (в частных случаях) ценой незначительных погрешностей, удаётся существенно упростить исходную задачу, снизить её размерность и на порядки снизить время расчётов. В частности, уменьшение размерности решаемой задачи может быть достигнуто, если вместо прямых методов, опирающихся на численные процедуры (метод конечных элементов, метод граничных элементов, метод сеток и др.), использовать идеи декомпозиции и агрегирования [8-11]. Во многих задачах применение метода декомпозиции (подконструкций) позволяет выполнить распараллеливание процессов вычислений.

Для обобщения метода расчёта релаксации остаточных напряжений в поверхностно упрочнённом слое плоских плит при ползучести, описанного в работе [3], на случай криволинейных стержней переменного сечения, в настоящей работе также применяется метод декомпозиции конструкции, который, с одной стороны, ценой незначительных упрощений снижает размерность задачи, а, с другой стороны, существенно сокращает время расчёта за счёт возможности распараллеливания вычислений.

Методика решения первой задачи - восстановления начального напряжённо-деформированного состояния в поверхностно упрочнённом слое по одной экспериментально замеренной компоненте тензора остаточных напряжений по толщине слоя - в настоящей работе не приводится, так как она полностью описана в монографии [3] и здесь используется без изменений.

Как и в работах [1-3], в настоящей работе также предполагается, что поверхностно упрочнённый слой является достаточно тонким (является «наклеенным» на «тело» стержня), не влияет на прочность стержня и деформируется в режиме «жёсткого» нагружения при заданном законе изменения тензора деформаций на поверхности стержня, который определяется из решения краевой задачи для криволинейного стержня, как если бы упрочнённого слоя не было.

Для решения поставленной задачи предлагается следующая упрощённая схема расчёта. Считается, что лопатка ГТД геометрически представляет собой стержень переменного сечения с малой пространственной кривизной оси. Семейством плоскостей, перпендикулярных оси, вдоль которой действуют центробежные массовые силы, лопатка разбивается на ряд стержней постоянного сечения (см. рис. 2). Однако для сведения этой задачи к плоской задаче, описанной в работах $[3,4]$, чисто геометрических рассуждений недостаточно.

При вращении стержней переменного сечения, в стержне создаётся не одномерное напря- 

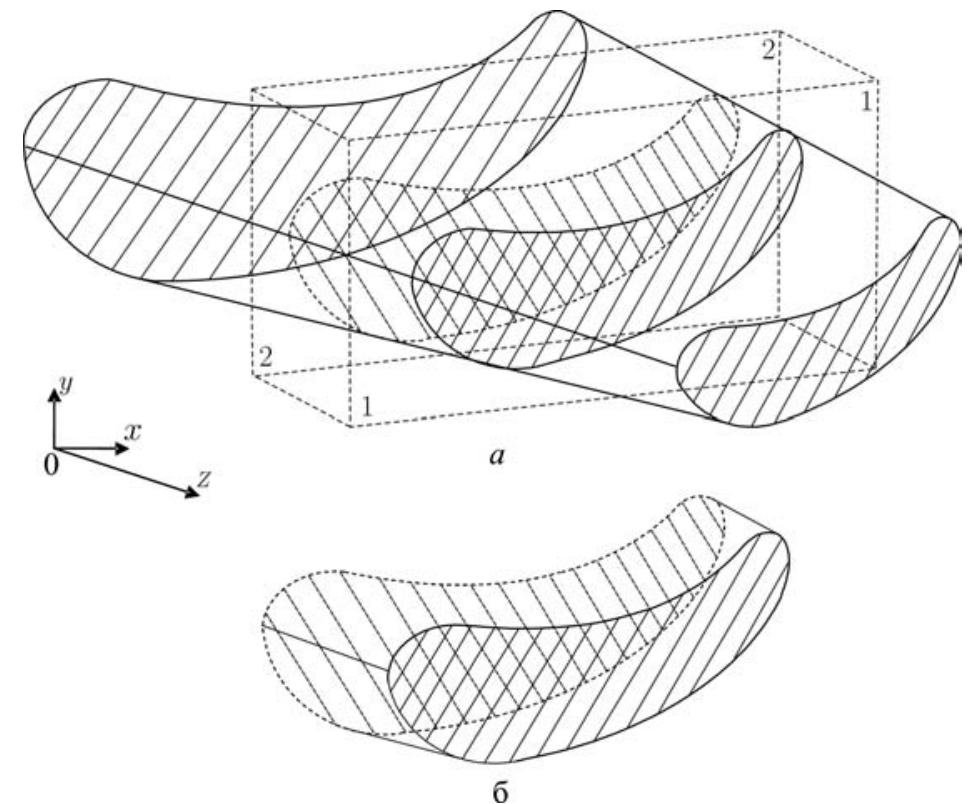

Р и с. 2. Схема декомпозиции лопатки на составной стержень с кусочно-постоянной площадью поперечного сечения

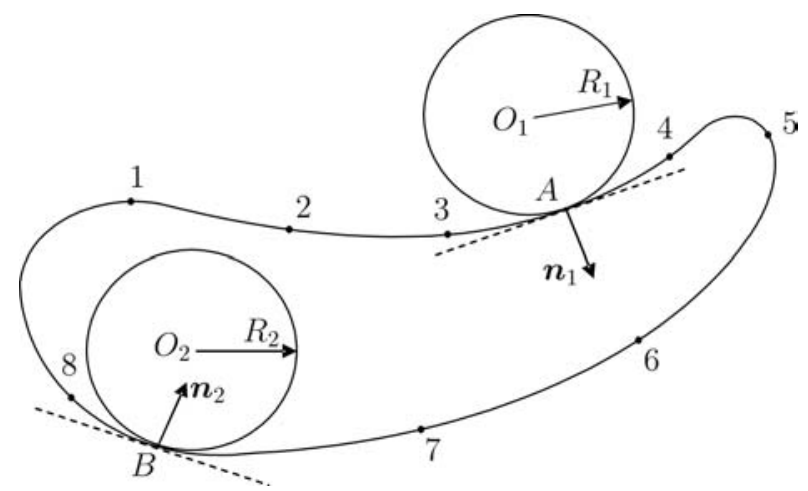

Р и с. 3. К расчёту релаксации остаточных напряжений на криволинейной поверхности жённое состояние, как в стержнях постоянного сечения [4], а сложное напряжённое состояние, при этом на поверхности стержня переменного сечения возникают касательные напряжения и деформации сдвига. Для учёта этого эффекта в стержнях переменного сечения предлагается следующая упрощённая схема расчёта кинетики остаточных напряжений в поверхностно упрочнённом слое в условиях ползучести.

1. Выполняется расчёт напряжённо-деформированного состояния стержня переменного сечения в поле массовых центробежных сил без учёта поверхностно упрочнённого слоя (например, методом конечных элементов) и определяется тензор деформаций $\varepsilon_{i j}^{1}$ в каждой интересующей точке поверхности в декартовой системе координат $0 x y z$.

2. Выполняется замена каждого элементарного стержня переменного сечения, ограниченного плоскостями 1-1 и 2-2 (рис. 2, б), на эквивалентный стержень постоянного сечения в поле массовых центробежных сил.

3. В каждом сечении эквивалентного стержня в зависимости от выпуклости или вогнутости вводится локальная цилиндрическая система координат с началом в центре соприкасающейся окружности (рис. 3) и осуществляется пересчет деформаций $\varepsilon_{i j}^{2}$ в цилиндриче-

ской системе координат по известным из расчёта деформациям $\varepsilon_{i j}^{1}$ в декартовой системе координат для всех необходимых точек поверхности.

4. Осуществляется расчёт на выпуклой поверхности сечения (точка $B$ ) как для упрочнённого цилиндра $[3,4]$, а на вогнутой поверхности как для упрочнённого кругового концентратора бесконечной плиты по методике, изложенной в работе [4].

На основании введённых гипотез тензор полной деформации $\varepsilon_{i j}$ в поверхностно упрочнённом слое можно представить в виде (условие «склейки» краевых задач):

$$
\varepsilon_{i j}(t)+\varepsilon_{i j}^{0}(r)=q_{i j}(r)+e_{i j}^{\text {res }}(r, t)+p_{i j}^{\text {res }}(r, t)+e_{i j}^{p \text { res }}(r, t) \quad(i, j=r, \theta, z) .
$$

Здесь $\varepsilon_{i j}(t)$ - тензор полной деформации на поверхности лопатки, определяемый из решения краевой задачи о её напряжённо-деформированном состоянии; $\varepsilon_{i j}^{0}(r), q_{i j}(r)-$ тензоры полных и остаточных пластических деформаций в поверхностно упрочнённом слое после процедуры ППД; $e_{i j}^{\text {res }}(r, t), p_{i j}^{\text {res }}(r, t)$ и $e_{i j}^{\text {pres }}(r, t)$ - тензоры упругой деформации, деформации ползучести и пластичной деформации в упрочнённом слое, рассчитываемые согласно схеме сложного напряжённого состояния через напряжения $\sigma_{i j}^{\text {res }}(r, t)$ в поверхностном слое. 
Выражая главные компоненты $e_{r}^{\text {res }}, e_{z}^{\text {res }}, e_{\theta}^{\text {res }}$ тензора упругой деформации через главные напряжения $\sigma_{r}^{\text {res }}, \sigma_{z}^{\text {res }}, \sigma_{\theta}^{\text {res }}$ в поверхностном слое по закону Гука, из (1) получаем

$$
\left\{\begin{array}{l}
\sigma_{r}^{\mathrm{res}}(r, t)-v \sigma_{z}^{\mathrm{res}}(r, t)-v \sigma_{\theta}^{\mathrm{res}}(r, t)=E\left[g_{r}(r, t)-f_{r}(r, t)\right], \\
-v \sigma_{r}^{\mathrm{res}}(r, t)+\sigma_{z}^{\mathrm{res}}(r, t)-v \sigma_{\theta}^{\mathrm{res}}(r, t)=E\left[g_{z}(r, t)-f_{z}(r, t)\right], \\
-v \sigma_{r}^{\mathrm{res}}(r, t)-v \sigma_{z}^{\mathrm{res}}(r, t)+\sigma_{\theta}^{\mathrm{res}}(r, t)=E\left[g_{\theta}(r, t)-f_{\theta}(r, t)\right],
\end{array}\right.
$$

откуда

$$
\sigma_{i}^{\mathrm{res}}(r, t)=\frac{A_{i}(r, t)}{1+v}+v \frac{A_{r}(r, t)+A_{z}(r, t)+A_{\theta}(r, t)}{(1-2 v)(1+v)},
$$

где введены функции $A_{i}(r, t)=E\left[g_{i}(r, t)-f_{i}(r, t)\right], \quad g_{i}(r, t)=\varepsilon_{i}(t)+\varepsilon_{i}^{0}(r)-q_{i}(r), \quad f_{i}(r, t)=$ $=p_{i}^{\text {res }}(r, t)+e_{i}^{p \text { res }}(r, t)(i, j=r, \theta, z)$.

Учитывая, что после процедуры упрочнения начальные пластические сдвиговые деформации $q_{i j}(r)=0 \quad(i \neq j)$, а упругие касательные деформации $e_{i j}^{\text {res }}$ связаны с касательными напряжениями $\sigma_{i j}^{\text {res }}$ законом Гука

$$
e_{i j}^{\mathrm{res}}=\frac{\sigma_{i j}^{\mathrm{res}}}{2 G}(i \neq j),
$$

где $G$ - модуль сдвига, связанный с модулем упругости $E$ и коэффициентом Пуассона $v$ coотношением $2 G=\frac{E}{1+v}$, из (1) получаем следующие соотношения:

$$
\sigma_{i j}^{\mathrm{res}}(r, t)=\frac{E}{1+v}\left[\varepsilon_{i j}(t)-\varepsilon_{i j}^{0}(r)-p_{i j}^{\mathrm{res}}(r, t)-e_{i j}^{p \mathrm{res}}(r, t)\right](i \neq j ; i, j=r, \theta, z) .
$$

Соотношения (2), (3) позволяют следить за процессом релаксации остаточных напряжений в упрочнённом слое при ползучести.

Однако для стержней переменного сечения с небольшим градиентом изменения площади величина касательных напряжений и соответствующих им сдвиговых деформаций незначительна и во многих случаях в расчётах ими можно пренебречь.

2. Решение задачи о напряжённо-деформированном состоянии лопатки при ползучести. Данная задача является второй в общей методике расчёта релаксации остаточных напряжений в поверхностно упрочнённом слое вращающихся стержней переменного сечения при ползучести. Решение этой задачи осуществляется на основе метода конечных элементов в пакете ANSYS и состоит из следующих этапов: 1) пространственное моделирование объёма лопатки; 2) конечно-элементное разбиение лопатки; 3) решение краевой задачи о напряжённодеформированном состоянии вращающейся лопатки в условиях ползучести; 4) анализ напряжённо-деформированного состояния по длине и периметру лопатки в условиях ползучести. Задача решена для всех трёх типов лопаток, представленных на рис. 1.

Следует отметить, что у всех лопаток на рис. 1 одинаковое корневое сечение; лопатка на рис. 1, $a$ получена параллельным переносом корневого сечения вдоль оси $0 z$; на рис. 1,6 представлена лопатка с переменной площадью поперечного сечения; на рис. 1, в лопатка с переменной площадью поперечного сечения имеет угол поворота хвостового сечения относительно корневого на $18^{\circ}$.

Для разбиения объёма лопатки на конечные элементы использован 20-ти узловой конечный элемент SOLID95 в форме тетраэдра (Tetrahedral Option), позволяющий проводить расчет НДС с учётом ползучести. Разбивание проводилось в автоматическом режиме на несколько десятков тысяч элементов. Одно из полученных разбиений для ра-

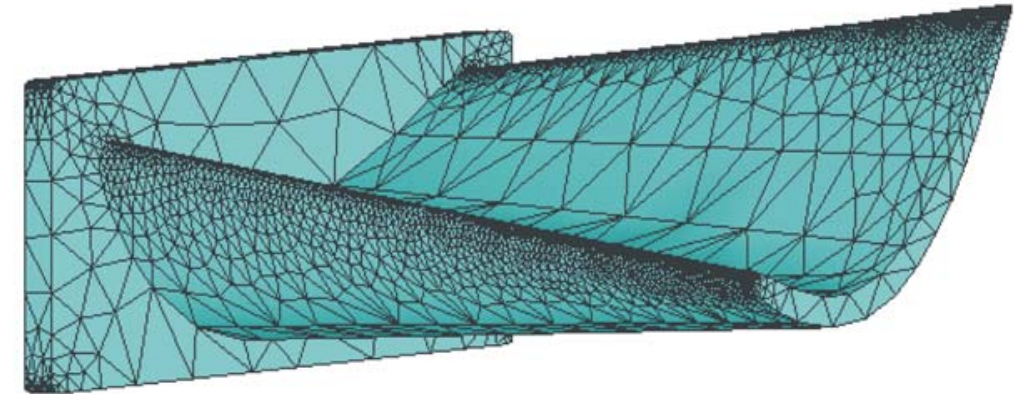

Рис. 4. Конечноэлементное разбиение закрученной лопатки переменного сечения (11 000 эелементов) 
бочей лопатки первой ступени турбины высокого давления газотурбинного комплекса ГТК 10 4 приведено на рис. 4 (без замка). На рис. 5 представлены 4 сечения этой лопатки ( $a-$ корневое сечение; $\sigma, 6$ - сечения, отстоящие на расстоянии $1 / 3$ и $2 / 3$ длины лопатки от корня; и $2-$ хвостовое сечение) с «характерными» точками поверхности, в которых исследуется напряжённо-деформированное состояние лопатки и проводится расчёт релаксации остаточных напряжений. Длина лопатки - 150 мм.

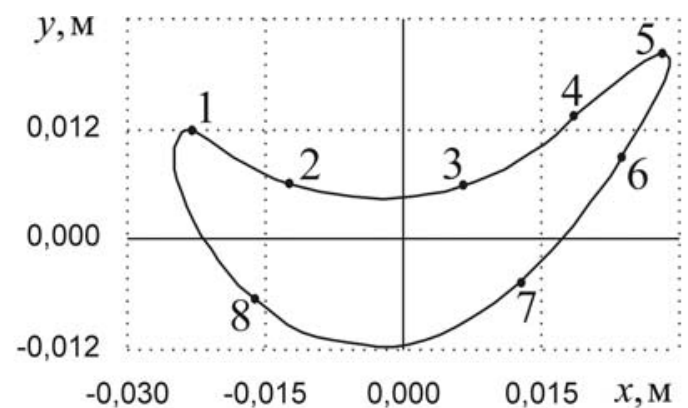

$a$

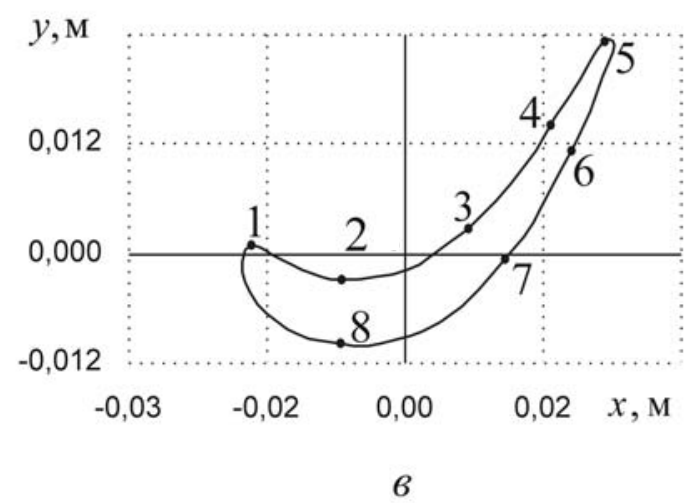

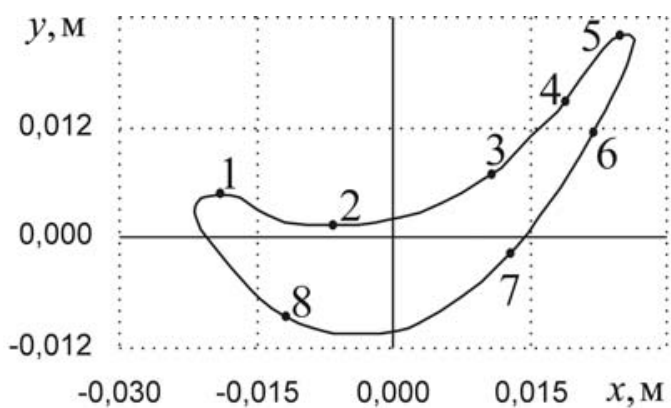

6

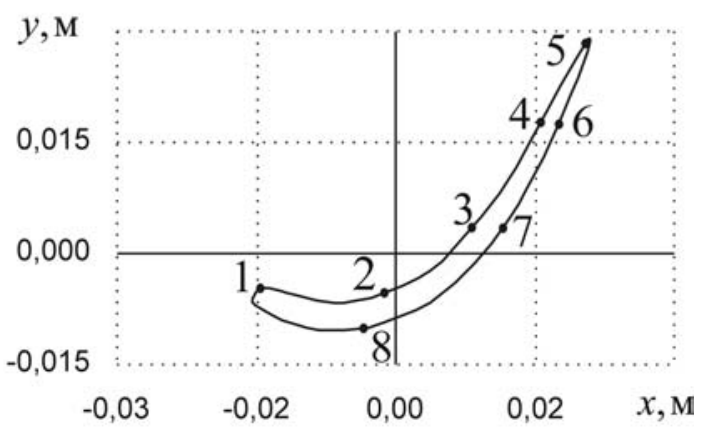

2

Рис. 5. Корневое ( $a$ ), средние (б и в) и хвостовое (2) сечения закрученной лопатки: цифры «характерные» точки поверхности лопатки

При расчётах для всех трёх лопаток в качестве модельного материала использовался сплав ЖС6КП. Расчёт осуществлялся для температуры $T=1000^{\circ} \mathrm{C}$ при числе оборотов $\omega=450$ рад/сек $\omega=350$ рад/сек и $\omega=270$ рад/сек. Свойства материала: $\rho=7850 \mathrm{\kappa} / \mathrm{M}^{3}$, $E=1,29 \cdot 10^{6}$ МПа, $v=0,3$, где $\rho-$ плотность материала; $E-$ модуль Юнга; $v-$ коэффициент Пуассона. Для решения задачи реологии была выбрана теория установившейся ползучести с законом

$$
\dot{w}_{i j}=c(S)^{m-1}\left(\frac{3}{2} \sigma_{i j}-\frac{1}{2} \sigma_{k k} \delta_{i j}\right),
$$

где $\dot{w}_{i j}$ - скорость деформации ползучести; $\sigma_{i j}$ - компоненты цензора напряжений; $S$ - интенсивность тензора напряжений, $\delta_{i j}-$ символ Кронекера; $c, m-$ константы модели (для сплава ЖС6КП при $\left.T=1000{ }^{\circ} \mathrm{C}-c=5,7 \cdot 10^{-33} ; m=3,11\right)$.

На рис. 6, 7 приведены кривые деформирования (для полной деформации) в некоторых «характерных» точках на поверхности. Приведённая информация используется в пункте 3 упрощённой схемы расчёта кинетики остаточных напряжений в поверхностно упрочнённом слое лопатки.

Результаты решения краевой задачи ползучести для рассматриваемых лопаток в поле центробежных сил позволил сделать следующие выводы [12]:

1) решение для лопатки, изображённой на рис. $1, a$, на расстоянии $\approx 50$ мм от корневого сечения по одномерной и трёхмерной моделям $[4,12]$ практически не отличаются; 

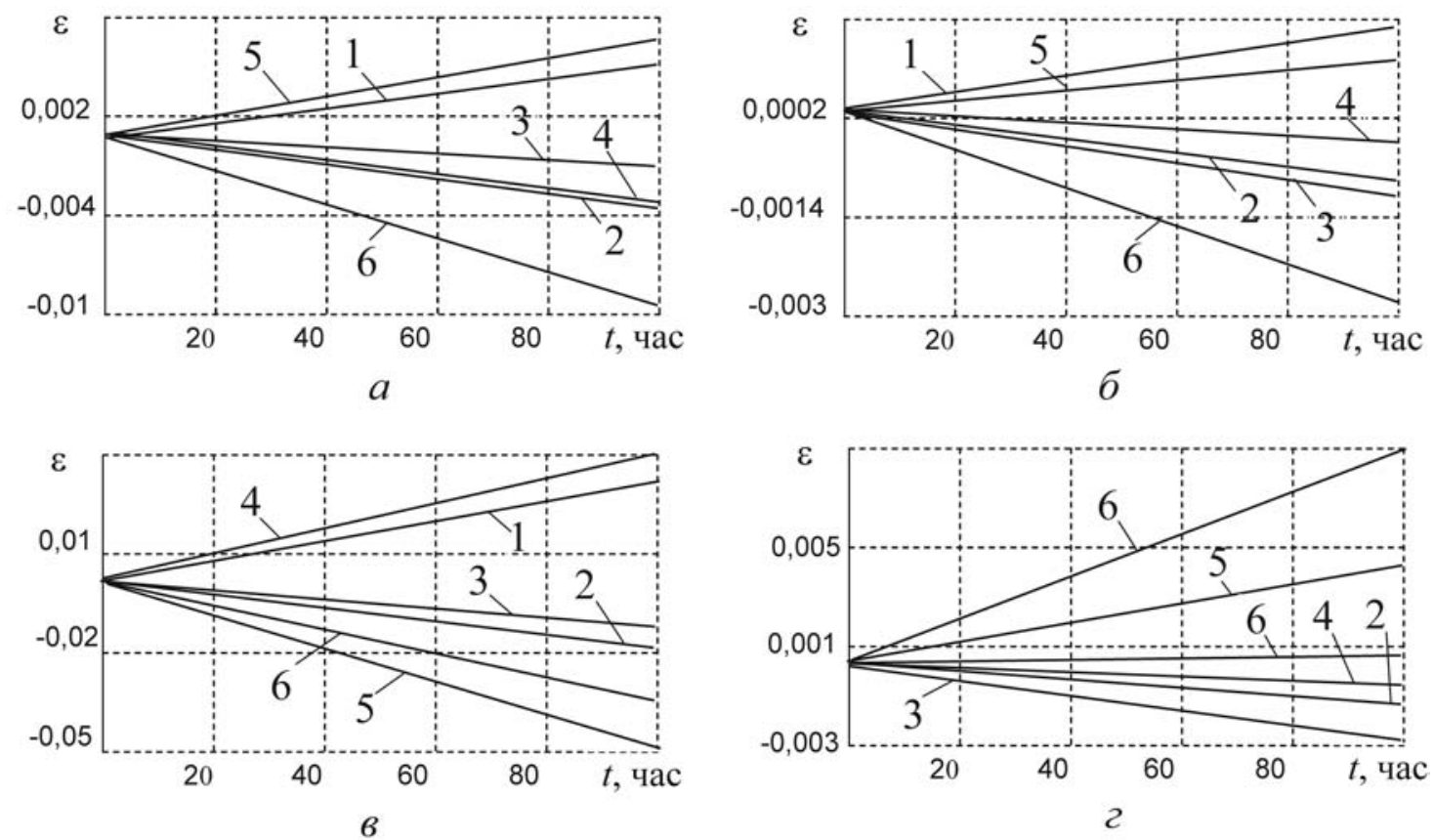

Рис. 6. Кривые деформирования в точках $1(a), 3($ б), 5 (в), 7 (2) корневого сечения для закрученной лопатки из сплава ЖС6КП $\left(T=1000{ }^{\circ} C\right)$ при числе оборотов $\omega=450$ рад/сек. Цифры: $1-\varepsilon_{z} ; 2-\varepsilon_{x} ; 3-\varepsilon_{y} ; 4-\varepsilon_{x y} ; 5-\varepsilon_{x z} ; 6-\varepsilon_{y z}$
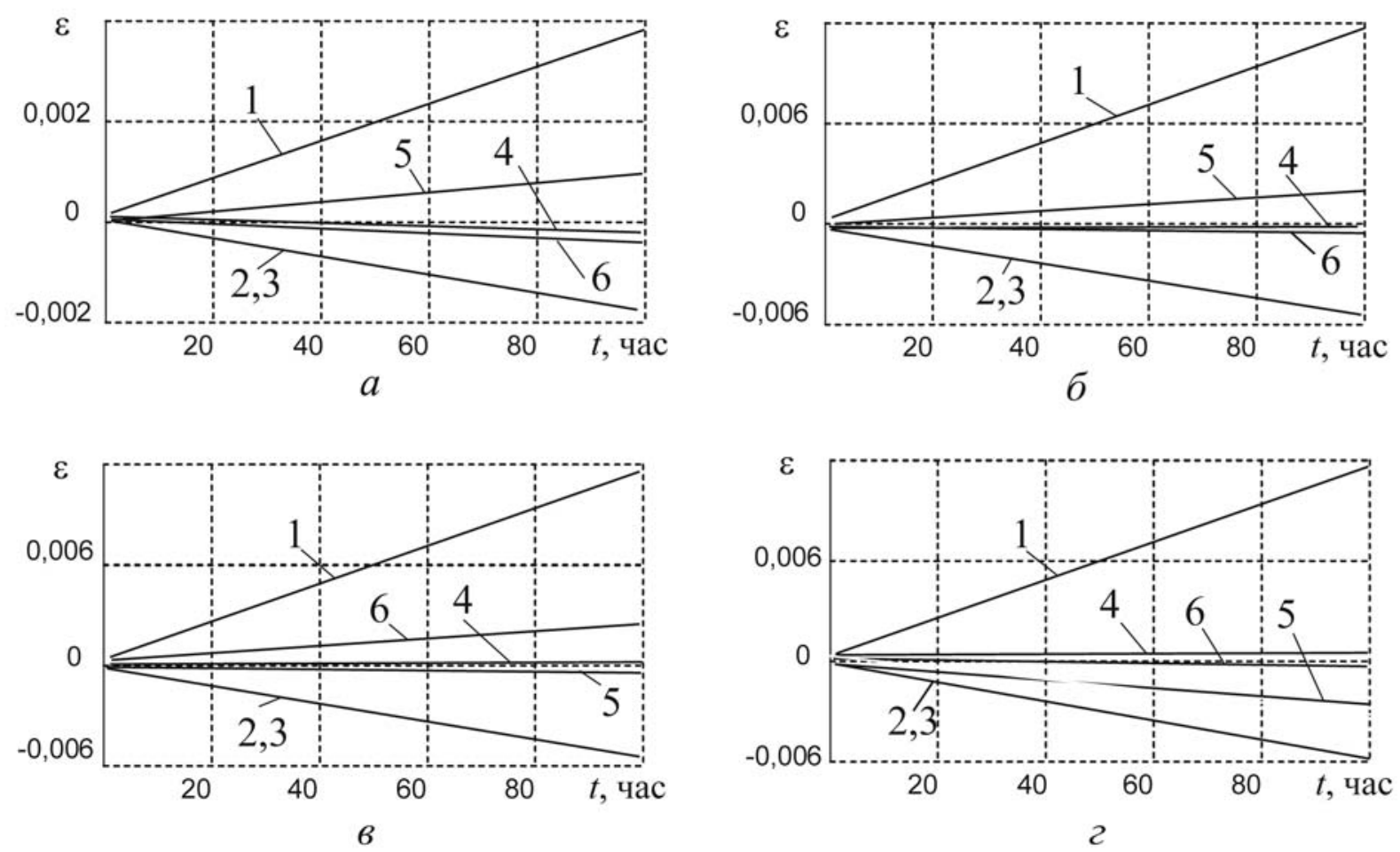

Рис. 7. Кривые деформирования в точках 1 (a), 3 (б), 5 (в), 7 (2) второго сечения для закрученной лопатки из сплава ЖС6КП $\left(T=1000{ }^{\circ} C\right)$ при числе оборотов $\omega=450$ рад/сек. Цифры: $1-\varepsilon_{z}$;

$$
2-\varepsilon_{x} ; 3-\varepsilon_{y} ; 4-\varepsilon_{x y} ; 5-\varepsilon_{x z} ; 6-\varepsilon_{y z}
$$

2) неоднородное напряженное состояние по периметру сечения лопаток также реализуется лишь в корневом сечении и области, примыкающей к нему. При этом уже на расстоянии $\approx 50$ мм от корневого сечения компоненты напряжений $\sigma_{x}, \sigma_{y}, \sigma_{x y}, \sigma_{x z}$ и $\sigma_{y z}$ много меньше (на два и более порядка для лопатки 1, $a$ и на один-два порядка для лопаток на рис. $1, \sigma, \varepsilon)$ величины осевых напряжений; 
3) осевая компонента напряжений $\sigma_{z}$, как в упругой области, так и области ползучести на порядок и более превосходит остальные компоненты тензора напряжений для всех сечений, удалённых на характерный размер от корневого сечения;

4) распределение напряжений по периметру сечения лопатки крайне неоднородно и величины напряжений, например $\sigma_{z}$, могут отличаться друг от друга более чем в 2 раза;

5) во всех сечениях (кроме корневого) происходит незначительный процесс перераспределения напряжений от упругого состояния до состояния установившейся ползучести с течением времени.

6) для пространственно закрученной лопатки (рис. 1, в) наблюдаются бо́льшие величины компонент тензора напряжений по сравнению с прямолинейной лопаткой, что, по-видимому, объясняется появлением изгибных дополнительных напряжений.

3. Расчёт релаксации остаточных напряжений в поверхностно упрочнённом слое вращающейся лопатки переменного сечения при ползучести. Целью данного пункта является иллюстрация предложенного в пункте 1 метода расчёта напряжённо-деформированного состояния в поверхностно упрочнённом слое вращающихся (в общем случае пространственно закрученных) лопаток переменного сечения при ползучести. Согласно этому методу лопатка переменного сечения аппроксимируется лопаткой кусочнопостоянного сечения, а далее решается основная задача для каждого сечения, состоящая из двух этапов:

1) находится напряжённо-деформированное состояние вращающейся лопатки в условиях ползучести в глобальной системе координат 0xyz одним из численных методов с последующим пересчетом тензора деформаций в локальной цилиндрической системе координат;

2) осуществляется расчёт кинетики остаточных напряжений в поверхностно упрочнённом слое лопатки (при её аппроксимации стержнями кусочно-постоянного сечения), который деформируется в режиме «жёсткого» нагружения при заданных значениях деформаций ползучести, которые получены на первом этапе решения задачи (в пункте 2), при этом на выпуклой поверхности сечения расчёт осуществляется как для кругового цилиндра [1-4], а на вогнутой как для цилиндрического кругового концентратора плиты [3].

Рассмотрим четыре сечения этой лопатки: нулевое, отстоящее на расстоянии 5 мм от корневого; второе, третье и четвёртое, отстоящие от корневого через 50 мм.

На первом этапе в четырёх сечениях лопатки в фиксированных выбранных восьми точках по её периметру, определяется центр соприкасающейся окружности, с которым связывается локальная цилиндрическая система координат. На рис. 8 показан профиль второго сечения с «характерными» точками 3,8 и соприкасающимися окружностями в глобальной системе координат $0 x y z$.

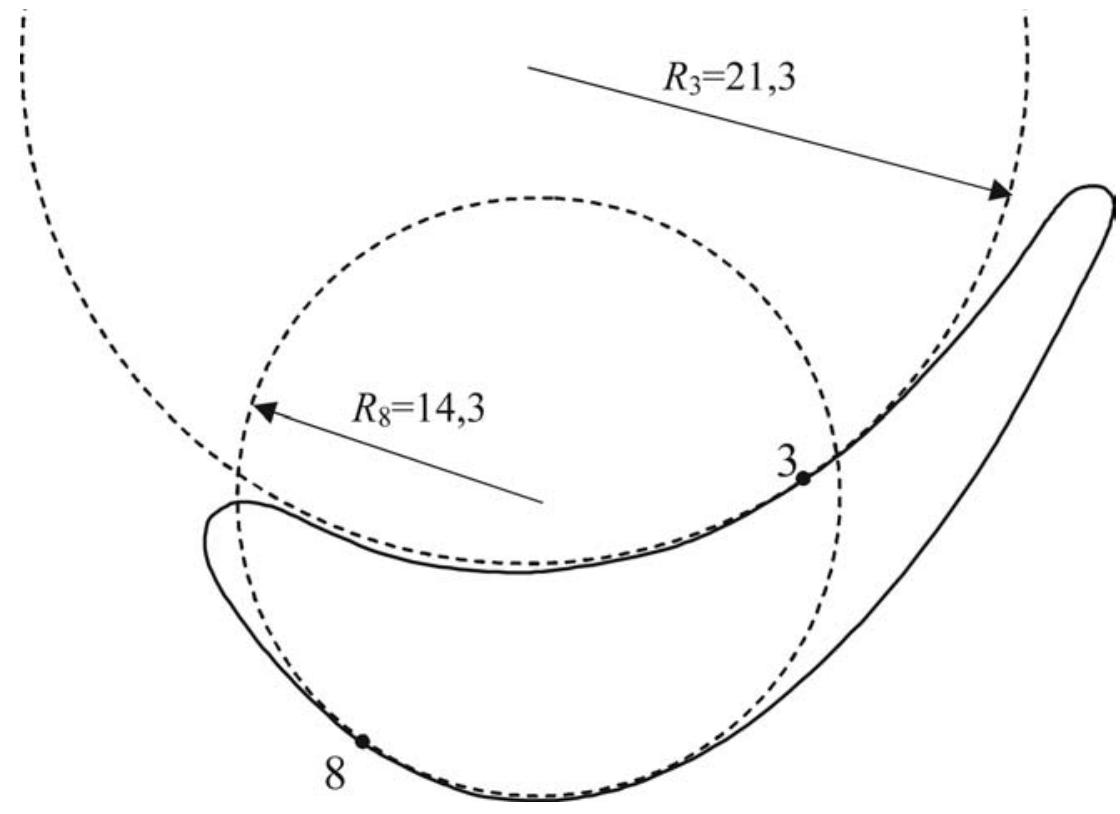

Рис. 8. Профиль 2 сечения закрученной лопатки и радиусы соприкасающихся окружностей (в миллиметрах) для точек 3 и 8

Поскольку компоненты тензора деформаций при ползучести получены в глобальной декартовой системе координат $0 x y z$, то производится перерасчёт компонентов тензора деформаций $\varepsilon_{i j}^{1}$ из декартовой системы координат в компоненты тензора деформаций $\varepsilon_{i j}^{2}$ локальной цилиндрической системы координат с её началом в центре соприкасающейся окружности, при этом ось $0 z$ является общей в той и другой системе. Эта процедура осуществляется в два этапа. На первом этапе осуществляется поворот декартовой системы координат (для каждой из восьми точек) в плоскости $0 x y$, таким образом, чтобы ось $0 x^{\prime}$ в новой 
декартовой системе координат $0 x^{\prime} y^{\prime}$ совпала с направлением $0 r$ в локальной цилиндрической системе координат. Тогда деформации $\varepsilon_{i j}^{\prime}$ в системе координат $0 x^{\prime} y^{\prime} z$ вычисляются с помощью формулы

$$
\varepsilon_{m s}^{\prime}=a_{m i} a_{s j} \varepsilon_{i j}^{1}, \quad m, s, i, j=1,2,3,
$$

где $\varepsilon_{i j}^{1}$ и $\varepsilon^{\prime}$; тензоры деформаций в исходной (глобальной) и повернутой декартовых системах координат; $a_{i j}$ - направляющие косинусы между системами координат $0 x y z$ и $0 x^{\prime} y^{\prime} z$.

На втором этапе осуществляется пересчет деформаций ползучести из декартовой системы координат $0 x^{\prime} y^{\prime} z$ в цилиндрическую систему координат $0 r \theta z$ по следующим формулам [13]:

$$
\varepsilon_{r r}=\varepsilon_{11}^{\prime}, \quad \varepsilon_{\theta \theta}=\frac{1}{r_{k}^{2}} \varepsilon_{22}^{\prime}, \quad \varepsilon_{z z}=\varepsilon_{33}^{\prime}, \quad \varepsilon_{r \theta}=\frac{1}{r_{k}} \varepsilon_{12}^{\prime}, \quad \varepsilon_{\theta z}=\frac{1}{r_{k}} \varepsilon_{23}^{\prime}, \quad \varepsilon_{z r}=\varepsilon_{31}^{\prime},
$$

где $\varepsilon_{i j}(i, j=r, \theta, z)$ - деформации в локальной цилиндрической системе координат; $r_{k}-$ значения радиусов в рассчитываемых точках; $\varepsilon_{k l}(k, l=1,2,3$; индекс 1 соответствует направлению $\left.0 x^{\prime}, 2-0 y^{\prime}, 3-0 z\right)$.

Пересчитанные описанным выше способом деформации ползучести служат исходными данными для расчёта релаксации остаточных напряжений в локальной цилиндрической системе координат по формулам (3) в контрольных точках $1,6,7,8$, расположенных на выпуклых поверхностях лопатки (расчёт по модели кругового цилиндра), а также для точек 2, 3, 4, 5, расположенных на выпуклых поверхностях лопатки (расчёт по модели кругового цилиндрического концентратора в плите).

4. Результаты и выводы. По предложенной методике выполнен детальный анализ кинетики остаточных напряжений в упрочнённом слое как для незакрученной, так и для закрученной лопаток первой ступени ГТД высокого давления, выполненных из сплава ЖС6КП, длиной 150 мм при различных режимах нагружения: $\omega=450$ рад/сек, $\omega=350$ рад/сек и $\omega=270$ рад/сек при $T=1000{ }^{\circ} \mathrm{C}$. Задача решена в пределах установившейся ползучести. Начальная эпюра, по которой восстанавливалось остаточное напряжённо-деформированное состояние (первая задача) после процедуры упрочнения, считалась одинаковой по всей поверхности лопатки.

На рис. 9 приведена типичная картина кинетики осевого остаточного напряжения $\sigma_{z}^{\text {res }}$ на поверхности упрочнённого слоя по периметру закрученной лопатки из сплава ЖС6КП $\left(T=1000{ }^{\circ} \mathrm{C}\right)$ при числе оборотов $\omega=450$ рад/сек. Здесь номера точек соответствуют номерам
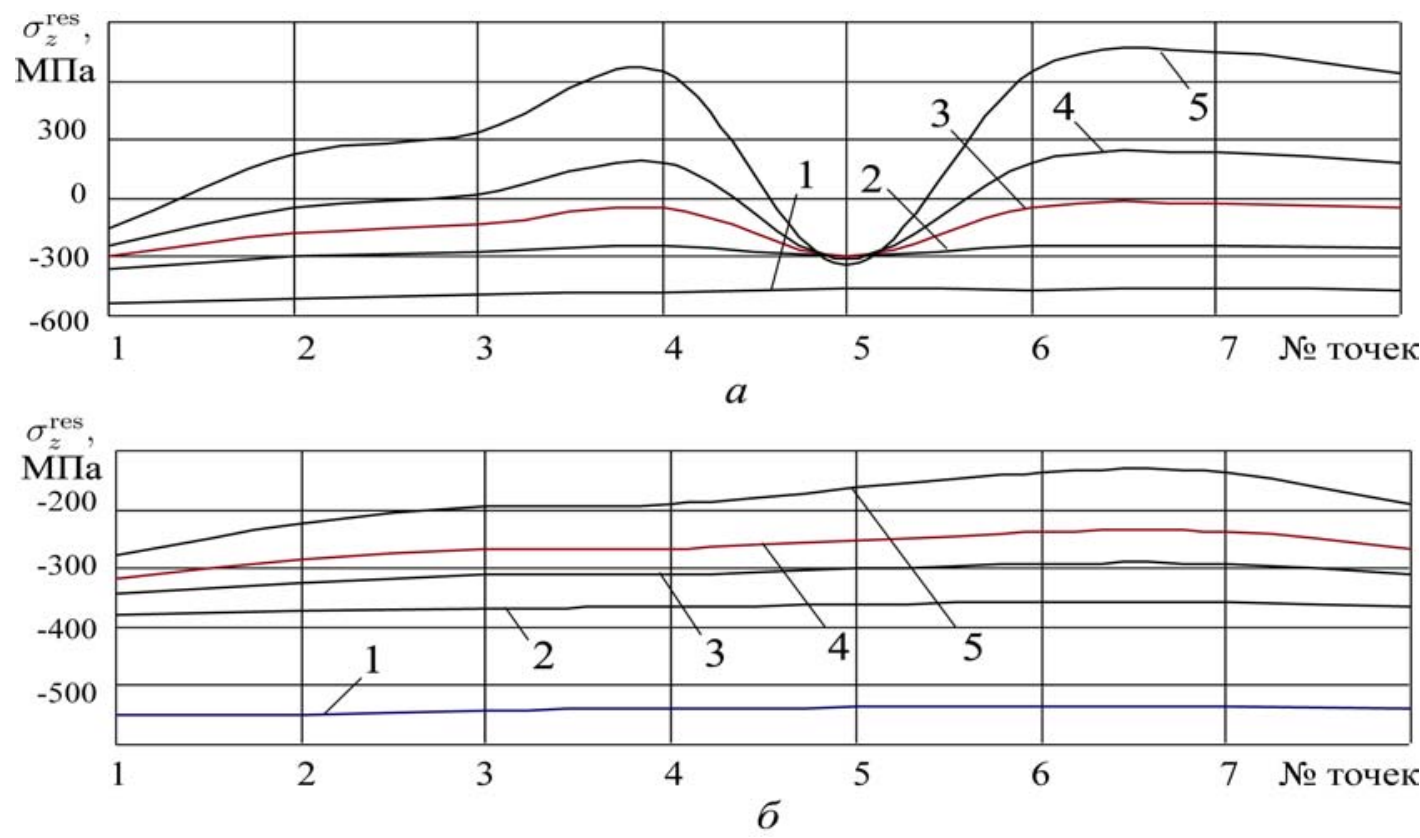

Рис. 3. Кинетика напряжений $\sigma_{z}^{\text {res }}$ на поверхности закрученной лопатки по периметру сечений: $a$ - отстоящего на 5 мм от корня; $\sigma$ - третье сечение, отстоящего на 100 мм от корня. Цифры: $1-t=0-0 ; 2-t=10 ; 3-t=50 ; 4-t=100 ; 5-t=200$ ч 
«характерных» точек. Ещё раз отметим, что после упрочнения остаточные напряжения по периметру сечения лопатки считались одинаковыми, что отражается кривой 1 на рис. 9, $a, \sigma$.

Сформулируем некоторые полученные результаты.

1. Релаксация напряжений протекает крайне неравномерно как по периметру любого сечения, так и по длине лопатки вдоль осевого направления. Процесс проходит интенсивнее в области корневого сечения.

2. В закрученной лопатке релаксация напряжений идёт интенсивнее, чем в незакрученной лопатке. Это, по-видимому, связано с тем, что центры тяжести сечений в закрученной лопатке оказываются смещёнными вдоль оси $0 z$ и поэтому возникают дополнительные изгибные напряжения.

\section{БИБЛИОГРАФИЧЕСКИЙ СПИСОК}

1. Радченко В. П., Саушкин М. Н. Расчёт релаксации остаточных напряжений в поверхностно упрочнённом слое цилиндрического изделия в условиях ползучести // Вестн. Сам. гос. техн. ун-та. Сер.: «Физ.-мат. науки», 2001. № 12. С. 61-72.

2. Радченко В. П., Саушкин М. Н. Математические модели восстановления и релаксации остаточных напряжений в поверхностно упрочнённом слое цилиндрических элементов конструкций при ползучести // Известия вузов. Машиностроение, 2004. № 11. С. 3-17.

3. Радченко В. П., Саушкин М. Н. Ползучесть и релаксация остаточных напряжений в упрочнённых конструкциях. М.: Машиностроение-1, 2005. 226 с.

4. Саушкин M. Н., Просвиркина E. А. Релаксация остаточных напряжений в поверхностно упрочнённом слое сплошного вращающегося цилиндра в условиях ползучести // В сб.: Мат. Моделирование и краевые задачи: Тр. Третьей Всерос. научн. конф. Т. 1: Математические модели механики, прочности и надёжности элементов конструкций. Самара: СамГТУ, 2006. С. 192-199.

5. Поздеев А. А., Няшин Ю. И., Трусов П. В. Остаточные напряжения: теория и приложения. М.: Наука, 1982. 109 c.

6. Стружанов В. В., Миронов В. И. Деформационное разупрочнение материала в элементах конструкций. Екатеринбург: УрО РАН, 1995. 192 с.

7. Стружанов В. В., Башуров Вяч. В. К определению полей собственных деформаций, инициирующих возникновение заданного поля остаточных напряжений // Актуальные проблемы современной науки: Тр. 5-ой Международн. конф. молодых учёных. Ч. 3, 4: Механика. Машиностроение и машиноведение. Металлургия. Литейное производство. Самара: СамГТУ. С. 63-66.

8. Первозванный, А. А., Гайц̧гори В. Г. Декомпозиция, агрегирования и приближенная оптимизация. М.: Наука, 1979. $344 \mathrm{c}$.

9. Никольский В. В., Никольская Т. Н. Декомпозиционный подход к задачам электродинамики. М.: Наука, 1983. $304 \mathrm{c}$.

10. Еремин Ю. А. Дискретное и континуальное агрегирование в конструкциях при ползучести // Теоретикоэкспериментальный метод исследования ползучести в конструкциях. Куйбышев: КуАИ, 1984. С. 41-56.

11. Клебанов Я. М., Давыљов А. Н. Многоуровневая декомпозиция конструкций методом аппроксимирующих моделей // Численные и аналитические методы расчета конструкций: Тр. международ. конф. Самара: СамГСА, 1998. C. 92-96.

12. Просвиркина E. A. Исследование эффекта Сен-Венана при растяжении лопатки вращающегося диска в поле центробежных сил // В сб.: Мат. Моделирование и краевые задачи: Тр. Третьей Всерос. научн. конф. Т. 1: Математические модели механики, прочности и надёжности элементов конструкций. Самара: СамГТУ, 2006. C. $172-176$.

13. Демидов С. П. Теория упругости. М.: Высшая школа, 1979. 432 с. 